\title{
Liderazgo empoderado como estrategia organizacional: revisión sistemática.
}

\section{Empowered leadership as an organizationa strategy: systematic review.}

Maria Teresa Reyes Chacón*, Luis Reyner López Arellanes**

Recibido: Marzo 2020

Aceptado: Junio 2020

Palabras Clave

Liderazgo empoderado, empoderamiento, revisión sistemática

\section{Keywords}

Empowering leadership, empowerment, systematic review.
El presente artículo es una revisión sistemática de literatura sobre liderazgo empoderado, que permita analizar la evolución del constructo de liderazgo empoderado dentro de las organizaciones, así como resultados en diferentes poblaciones y áreas del mercado. Adicionalmente se analizan las metodologías utilizadas y los abordajes teóricos concluyendo con propuesta para definición unificada y áreas de oportunidad para futuras investigaciones en las que destacan: los efectos menos positivos del mismo, seguimiento del liderazgo por un periodo de tiempo y los procesos propios de implementación con la temporalidad requerida, así como el estadio de desarrollo del equipo de trabajo donde se expresa.

Abstract

The present article is a systematic review of literature on empowered leadership that allows analyzing the evolution of the empowered leadership construct within organizations, as well as results in different populations and market areas. Additionally, the methodologies used and the theoretical approaches are analyzed, concluding with a proposal of unified definition and on the areas of opportunity for future research in which the following stand out: the less positive effects of the same, follow-up of the leadership for a period of time and the own processes of implementation with the required temporality, as well as the stage of development of the work team where it is expressed.

\section{Introducción}

El liderazgo ha demostrado su importancia en numerosas investigaciones (Furler, Cleland, Del Mar et al., 2008; Lega, Prenestini, y Rosso, 2017, Liu, 2015; McDermott, Kidney, y Flood, 2011 y Praszkier, 2015), desde los años 50 del siglo anterior es un tema recurrente de estudio dentro de las organizaciones, para describir y clasificar las diferentes tipologías de líderes (Baker, 2011; Enterkin, Robb, y Mclaren, 2013 y Zhang y Gheibi, 2015).

La evolución del liderazgo ocurre a la par que la de las organizaciones, volviéndose cada vez más complejo, actualmente no es suficiente un líder con carisma, autoridad, motivación, etc.(Ismail, Mohamed, Sulaiman, Mohamad, y Yusuf, 2011), se requiere un personaje capaz de transformar, servir y gestionar recursos de diferente índole; con habilidad para desarrollar nuevos líderes mediante la toma de decisiones compartida (Parris y Peachey, 2013), utilizando comunicación efectiva y empoderamiento que genere autogestión así como automotivación en los

* Candidata a Doctor en Ciencias Administrativas,. Maestra en Administraciónn, Coordinador médico de primer nivel en el Instituto Mexicano del Seguro Social. tesareyes@gmail.com

** Doctorante en Terapia enPpareja. Médico de primer nivel en el Instituto Mexicano del Seguro Social. luisreyner2007@hotmail.com 
seguidores (Amundsen y Martinsen, 2015; Wong Humborstad, Nerstad, y Dysvik, 2014).

El objetivo de la presente revisión es visualizar y analizar la evolución de los enfoques sobre liderazgo empoderado en las últimas dos décadas.

El liderazgo empoderado (LE) se separa como constructo de los demás tipos de liderazgo, al identificar desde su conceptualización implicaciones positivas en investigaciones realizadas específicamente sobre él (Ahearne, Mathieu, y Rapp, 2005; Ismail, Mohamed, Sulaiman et al., 2011). Se destacan características y asociaciones con otros constructos psicológicos dentro de las organizaciones, facilitando alcanzar objetivos programados, mediante diferentes vías en cada caso ( Lee, Willis, y Tian, 2017). Además, se ha descrito como un factor asociado que promueve tanto la creatividad como la innovación (Özarallı, 2015), incluso disminuye el cinismo organizacional mediante el empoderamiento psicológico (Lorinkova, Pearsall, y Carolina, 2013); pero sobre todo, no es específico a un tipo de organización o población: en empleados de trato directo al público de la industria según Hao, He y Long, (2017).

Amundsen \& Martinsen, (2014b) por su parte, proponen al LE cómo proceso que involucra tres aspectos: compartir el poder, la motivación y apoyo para el desarrollo. No obstante, se ha evidenciado el alcance del LE desde múltiples ópticas: Wong Humborstad et al., (2014) resaltan la importancia de concientizar sobre el nivel ejercido de liderazgo empoderado, recomiendan a los líderes ser constantes en la forma de compartir las responsabilidades para la toma de decisiones con sus subordinados, reduciendo así la incertidumbre operacional consecuente de un liderazgo poco claro. Por otro lado, Zhu y Chen, (2014); proponen un liderazgo empoderado centrado en el grupo, enfatizando el trabajar como una entidad para coordinar esfuerzos; así los miembros del equipo adoptan el trabajo como una norma subjetiva y generan una interacción positiva para colaborar y lograr objetivos comunes.

Para Kim y Beehr, (2017) los líderes empoderadores apoyan a los seguidores, proporcionan los recursos necesarios, comparten información relevante en la realización de las tareas y expresan confianza en las habilidades de los seguidores, llevándolos a

Cuadro 1: Revisiones previas: objetivos y temas

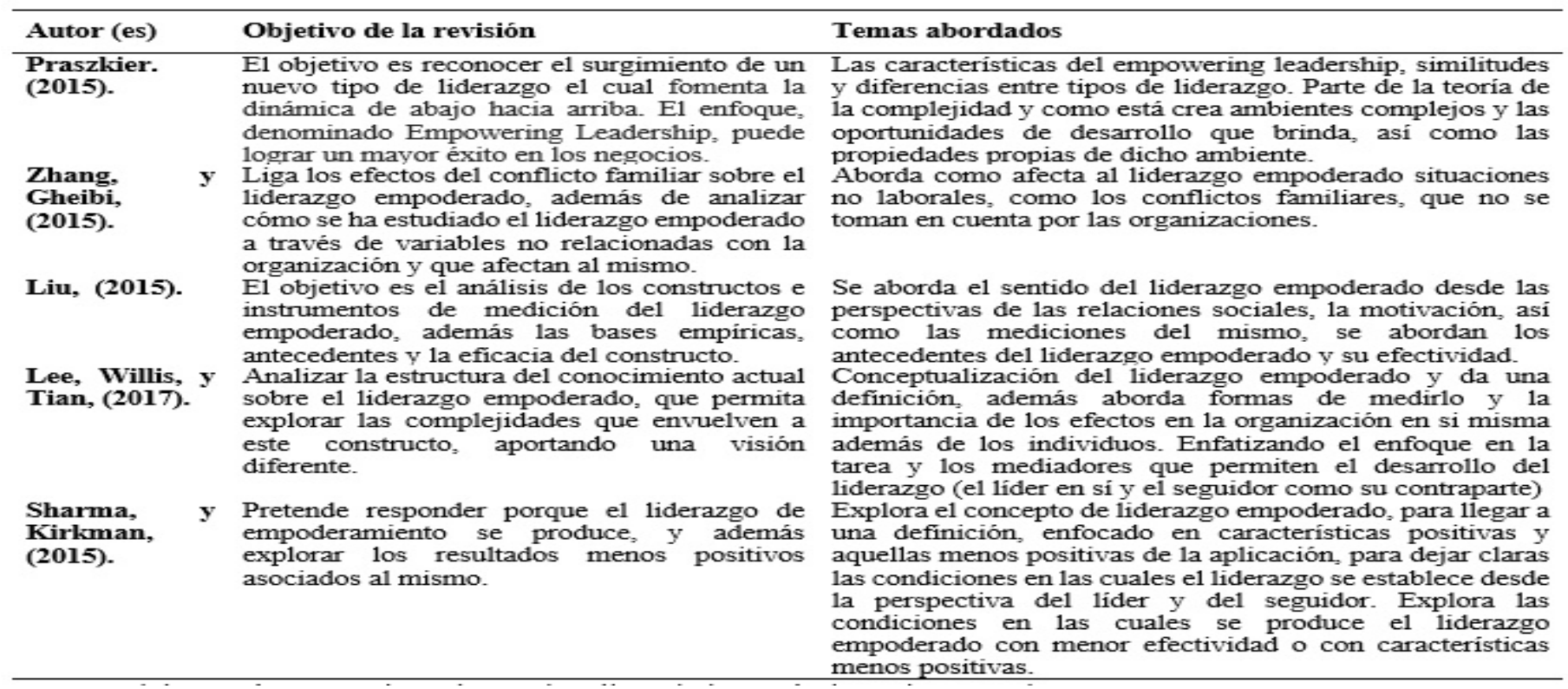

Fuente: elaboración propia basada en el análisis de los artículos

turística, mejora la relación laboral, motiva a los empleados a intercambiar ideas y experiencias que ayudan a llevar a buen término iniciativas que mejoran el desempeño del servicio hotelero ( $\mathrm{Wu}$ y Chen, 2015). Por su parte, en grupos de enfermeras él LE favorece el pensamiento sistémico y estratégico, aumenta la comunicación entre líderes y trabajadores maximizando la participación laboral, incrementa la afición al trabajo y el rendimiento, volviéndolos más leales y comprometidos (Abdul y Abbas, 2016), aun cuando implica al líder como al seguidor, la participación directa de los lideres tiene efectos diferenciales sobre el rendimiento laboral de los empleados un nivel alto de motivación y rendimiento.

No obstante se han descrito efectos desfavorables asociados al liderazgo empoderado, por ejemplo, desconocer si el comportamiento de los líderes de empoderamiento puede abrumar a los seguidores, lo cual destaca la importancia de ejercer el empoderamiento como realidad y no como ilusión, asevera Cheong, Spain, Yammarino,et al. (2016).

El estudio del Liderazgo Empoderado busca comprender, analizar y replicar conductas o ambientes de éxito, la revisión sistemática de este tema específicamente, permite visualizar Vol. 10 Núm. 18 Enero-junio 2020 21 
discrepancias entre las diferentes investigaciones, analizar las perspectivas y metodologías empleadas (Manterola, Astudillo, Arias et al 2013), a fin de integrar un esquema evolutivo en relación a los abordajes y de esta manera organizar los hallazgos y visualizar aspectos pendientes por indagar. Revisiones previas de la literatura se analizan y sintetizan en el cuadro 1

La presente revisión sistemática busca organizar los abordajes y la evolución del liderazgo empoderado (LE) que permita dar estructura y continuidad al constructo, además describir resultados obtenidos de la asociación del LE a diferentes constructos, visualizar los vacíos remanentes. Además describir las metodologías utilizadas para evaluar el liderazgo y los instrumentos empleados, a fin de establecer diferencias entre los aportes de dichas investigaciones; durante las últimas dos décadas.

¿Cuáles características son comunes o diferentes sobre él Liderazgo Empoderado en las investigaciones realizadas en las últimas dos décadas desde una perspectiva integradora?

\section{Metodología}

La actual revisión sistemática adopta la estructura que sugiere la guía Preferred Reporting Items for Systematic Review and Meta-Analyses (PRISMA)

Los estudios incluidos son empíricos, publicados en idioma inglés e incluidos en revistas con factor de impacto. Se efectuó una búsqueda sistemática de la literatura en las siguientes bases de datos: Web of Science, Emerald, ScienceDirect, Proquest, Redalyc, Springer, Google Scholar, y circunscribe un periodo de dos décadas
(1997 a 2017) realizada de febrero a septiembre de 2018. Se consideran múltiples tipos de poblaciones participantes, así como diferentes metodologías e instrumentos para evaluar el constructo del liderazgo empoderado.

La búsqueda se realiza utilizando la técnica booleana con las palabras clave "leadership empowerment" or "leadership empowering", incluidas en el título, dentro del periodo de 1997 a 2017.

Se analizan los contenidos a través del resumen, integrándolos en un formato especialmente diseñado para la extracción de los datos que permita organizar la información y las variables recabadas que son: objetivos, poblaciones estudiadas, metodología aplicada, instrumentos utilizados, factor de impacto, año de publicación.

Los principales sesgos entre estudios es la variabilidad entre los mismos, ya que el constructo de liderazgo empoderado se ha estudiado en múltiples poblaciones, diferentes nacionalidades, en los cinco continentes, con personas de diferente extracto social, económico y cultural, así como en diversas áreas laborales.

Por lo tanto, esta diversidad puede manifestarse en un Sesgo de publicación relacionada con la selección de resultados positivos del liderazgo empoderado.

\section{Resultados}

Inicialmente se realiza una búsqueda general de las palabras clave, encontrando 115 artículos útiles, posteriormente se

Cuadro 2: El proceso se siguió de la siguiente manera:

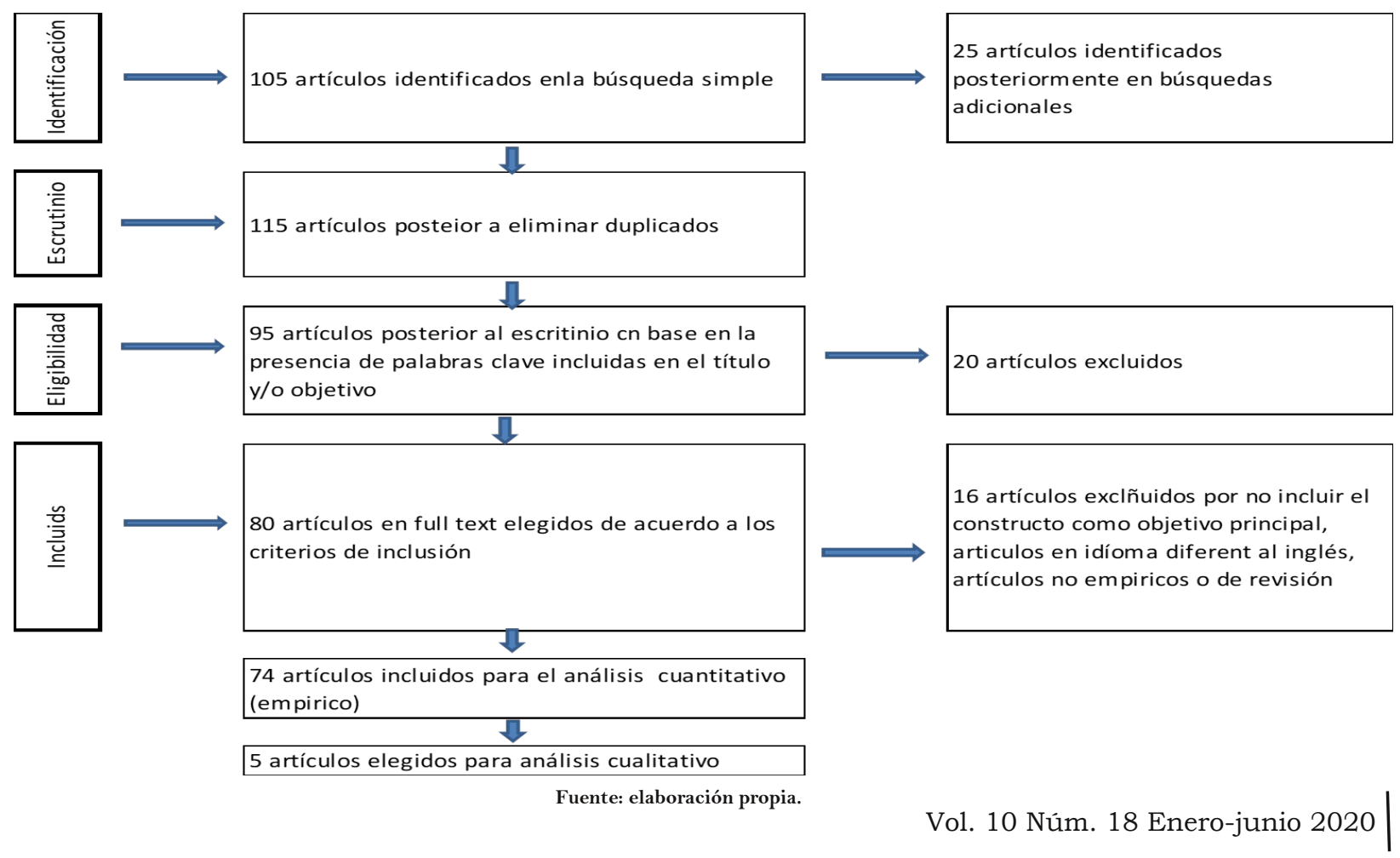


limita a la presencia de la palabra clave en el título y objetivo además de eliminar los duplicados dejando 79 artículos, en una tercera evaluación se suman 5 artículos de revisión que se eliminan del análisis cuantitativo, dejando finalmente 74 artículos para su análisis.

Para el proceso descriptivo de los datos del artículo se construyó un cuadro que contiene los artículos analizados, los cuales se ordenaron alfabéticamente y se les asignó un número secuencial como identificación, de esta manera se puede relacionar las características evaluadas a la referencia bibliográfica. (Véase anexo $1)$.

Los 74 artículos considerados para el análisis el 100\% están en idioma inglés, el $92 \%(\mathrm{n}=68)$ son estudios empíricos, el $89 \%$ $(n=65)$ de los artículos se encuentran en revistas con factor de impacto. Geográficamente la mayoría de estudios sobre liderazgo se realizan en el continente asiático (39\%), siendo América el último lugar con apenas un $11 \%$ incluyendo Estados Unidos (en el Cuadro 2 se muestran detalles de la distribución geográfica).

Cuadro 3. Distribución geográfica de las investigaciones.

\begin{tabular}{|l|l|l|}
\hline Área Geográfica & Número de artículos & Referencias \\
\hline Asia & $\mathrm{n}=29(39 \%)$ & $34,40,24,36,51,13,73,16,38,39,25,37,66$, \\
& & $69,21,71,9,61,29,28,60,62,22,68,33,32$, \\
& & $50,58,72$. \\
\hline Otros & $\mathrm{n}=18(24 \%)$ & $42,35,26,10,45,1,14,4,17,41,53,56,70$, \\
& & $74,55,2,3,47$. \\
\hline Europa & $\mathrm{n}=16(22 \%)$ & $\begin{array}{l}23,8,7,11,19,20,6,5,57,18,54,44,46,49, \\
65,12 .\end{array}$ \\
\hline América & $\mathrm{n}=11(15 \%)$ & $30,52,31,48,43,27,63,15,67,64,59$. \\
\hline
\end{tabular}

Fuente: creación propia de la tabla de análisis de artículos.

La elección de poblaciones a estudiar destaca la diada (líder-seguidor) con un $36 \%$ de los estudios donde se hace una evaluación en ambos lados del liderazgo, y la evaluación a empleados en puestos directivos (26\%) desde la perspectiva del líder. Las demás poblaciones estudiadas son diversas desde estudiantes, personal del sector salud, empleados de mostrador, etc., como se muestra en el cuadro 4.

Para las técnicas de recolección de datos la encuesta es la más utilizada con un $87 \%$ de los casos y de ellas la encuesta de aplicación directa ocupa el primer lugar con el $61 \%$ global, donde la empresa interviene y apoya al grupo investigador para la realización de la investigación, la encuesta on line toma relevancia con un $15 \%$. Por su parte las investigaciones con finalidad de validación de escalas se sitúan en segundo lugar de frecuencia con el $9 \%$ del total de la muestra y aquellos estudios con uso de simuladores y/o intervenciones únicamente se aplicaron en $4 \%$ de los casos. (Cuadro 4). Finalmente, el instrumento de evaluación se ha diversificado en la creación y modificación de escalas destacando 6 instrumentos como los de mayor utilización, la escala de leadership empowerment behaviour propuesta por Arnold, Arad, Rhoades et al., siendo la de uso más popular con un $31 \%$. (Cuadro 4)

Dentro de la diversidad de enfoques destaca además las múltiples asociaciones del LE con otros constructos psicológicos como se muestra en el cuadro 5 .

\section{Definición}

En el liderazgo empoderado el principal rasgo es compartir el poder, favorecer la motivación, autonomía y responsabilidad del seguidor, además mediante el coaching la adaptación al entorno, el desarrollo de la autoridad, la auto eficiencia, el compromiso afectivo entre líder y seguidor. Permite un intercambio activo de información, donde el seguidor desarrolla un papel activo en la toma de decisiones y trabajo en equipo, generando autosuficiencia, autocontrol, autogestión mediante una relación interpersonal positiva, que facilita procesos de cambio, mejora la significancia del trabajo, elimina el sentido de impotencia y los lleva a ser más receptivos ante la jerarquía.

Para fundamentar teóricamente similitudes y/o diferencias entre otros tipos de liderazgo contra Liderazgo Empoderado, se han empleado diferentes teorías, sin llegar a un consenso, Martin, Liao y Campbell, (2013), Sharma y Kirkman, (2015), Ozaralli, (2015) utilizan la teoría propia del liderazgo; por su parte, Amundsen y Martinsen, (2013) la teoría de la autodeterminación; Lorinkova, Pearsall y Sims, (2013) buscan en la teoría del liderazgo situacional y Hassan, Mahsud, Yukl y Prussia, (2013), Biemann, Kearney y Marggraf, (2015) en la teoría del intercambio entre los miembros. En los últimos años los fundamentos teóricos cambian, como ejemplo Auh, Menguc, y Jung, (2014) integran la teoría del empoderamiento; Li, He, Yam y Long (2015) la teoría de roles la teoría y finalmente Praszkier (2015) la teoría de la complejidad.

\section{Discusión}

El abordaje del liderazgo de empoderamiento de forma predominante se ha realizado utilizando la medición cuantitativa a través de una serie de parámetros preestablecidos contenidos en un cuestionario como evaluación única, dejando poco espacio para la investigación documental y prácticamente nada al enfoque cualitativo lo cual concuerda con los hallazgos de Liu (2015), como consecuencia de lo anterior se han generado una continua construcción y propuesta de instrumentos de medición o evaluación del constructo, sin embargo, destaca el escaso interés en la validación de instrumentos utilizados o propuestos en poblaciones o contextos diferentes; la evolución sobre este constructo se manifiesta principalmente en la integración de los individuos involucrados: de ser aplicado inicialmente desde una perspectiva unilateral (líder o 
seguidor) hacia compilar ambas perspectivas (líder y seguidor) además se ha buscado describir la perspectiva del líder en relación a cómo visualizar y reconocer ambientes propicios para el empoderamiento (Sharma y Kirkman, 2015), empero, las características específicas de dichos ambientes no se especifican ni se describen. Destaca un estudio donde revisan contextos externos a las organizaciones que afectan el desarrollo del liderazgo empoderado, sin embargo, evalúan únicamente el factor familiar, dejando

Cuadro 4. Poblaciones, técnicas y herramientas de investigación utilizadas.

\begin{tabular}{|c|c|c|c|}
\hline & Características & Frecuencia & Artículos (No. Asignado) \\
\hline \multirow{7}{*}{ 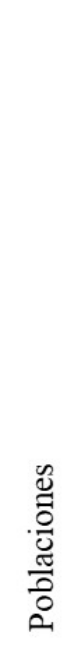 } & Diadas de líder/seguidor & $\begin{array}{l}36 \% \quad(\mathrm{n}= \\
27) \quad\end{array}$ & $\begin{array}{l}14,17,59,62,68,28,72,6,25,19, \\
20,21,37,2,5,24,61,42,43,58, \\
16,73,65,38,53,69,36 .\end{array}$ \\
\hline & $\begin{array}{l}\text { Empleados en puestos } \\
\text { directivos }\end{array}$ & $\begin{array}{l}26 \% \\
(\mathrm{n}=19)\end{array}$ & $\begin{array}{l}1,33,15,32,29,45,46,11,49,8, \\
34,13,39,3,35,41,47,51,52 .\end{array}$ \\
\hline & $\begin{array}{l}\text { Empleados de } \\
\text { directo al público }\end{array}$ & $12 \%(n=9)$ & $22,54,57,9,48,23,44,55,66$ \\
\hline & Estudiantes o docentes & $9 \%(n=7)$ & $50,67,64,27,63,56,71$ \\
\hline & $\begin{array}{l}\text { Empleados del sector } \\
\text { salud }\end{array}$ & $8 \%(n=6)$ & $4,12,70,40,26,74$ \\
\hline & $\begin{array}{l}\text { Empleados de la industria } \\
\text { manufacturera }\end{array}$ & $7 \%(n=5)$ & $7,10,30,52,31$ \\
\hline & $\begin{array}{l}\text { y trabajadores de } \\
\text { asociaciones religiosas }\end{array}$ & $3 \%(n=2)$. & 18,60 \\
\hline \multirow{5}{*}{ 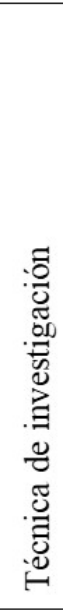 } & aplicación directa & $\begin{array}{l}61 \% \\
(\mathrm{n}=45)\end{array}$ & $\begin{array}{l}4,22,54,57,62,27,28,29,44,12, \\
18,72,46.9,19,25,7,37,21,69, \\
71,66.34,40,43,26,39,38,51,24, \\
36,58,16,13,65,11,5,23,35,41, \\
47,53,55,56,65,74 .\end{array}$ \\
\hline & aplicación on line & $\begin{array}{l}15 \%(\mathrm{n}= \\
11)\end{array}$ & $\begin{array}{l}14,32,33,59,64,17,68,60,49,6 \text {, } \\
20 .\end{array}$ \\
\hline & Iี & $11 \%(n=8)$ & $48,61,31,30,52,8,45,3$ \\
\hline & $\begin{array}{l}\text { Prueba y/o validación de } \\
\text { un instrumento de } \\
\text { evaluación o uso de } \\
\text { simulador }\end{array}$ & $9 \%(n=7)$ & $15,50,67,1,10,70,73$ \\
\hline & Con una intervención & $4 \%(n=3)$ & $2,42,63$ \\
\hline \multirow[b]{3}{*}{ 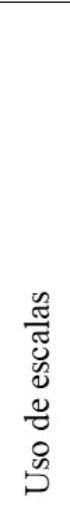 } & $\begin{array}{l}\text { Uso de la escala de } \\
\text { leadership empowerment } \\
\text { behaviour propuesta por } \\
\text { Arnold et al. }\end{array}$ & $\begin{array}{l}31 \% \\
(\mathrm{n}=23)\end{array}$ & $\begin{array}{l}14,17,22,32,50,64,67,19,21,27, \\
66,36,38,30,31,46,29,24,42,54, \\
55,70,74 .\end{array}$ \\
\hline & $\begin{array}{l}\text { Uso de la escala } \\
\text { desarrollada por } \\
\text { et al. de } \\
\text { 12-item }\end{array}$ & $\begin{array}{l}23 \% \\
(\mathrm{n}=17)\end{array}$ & $\begin{array}{l}33,62,68,28,43,12,72,37,61,71, \\
65,13,16,34,39,8,1 .\end{array}$ \\
\hline & $\begin{array}{l}\text { The Leader Empowering } \\
\text { Behaviour Questionnaire } \\
\text { (LEBQ) de Konczak et al., } \\
2000\end{array}$ & $11 \%(n=8)$ & $18,10,48,41,49,20,26,56$ \\
\hline
\end{tabular}

Fuente: creación propia de la tabla de análisis de artículos. 
Cuadro 5. Constructos psicológicos asociados al Liderazgo Empoderado.

\begin{tabular}{|c|c|c|}
\hline & $\begin{array}{l}\text { Constructo asociado al Liderazgo } \\
\text { Empoderado }\end{array}$ & artículos \\
\hline \multirow[t]{3}{*}{ 安 } & Empoderamiento Psicológico & $\begin{array}{l}17,68,54,4,6,9,21,31,48,49,40, \\
34,42\end{array}$ \\
\hline & Coaching & $1,22,50,52$ \\
\hline & Satisfacción Laboral & $69,5,7,47,23$. \\
\hline \multirow{11}{*}{ 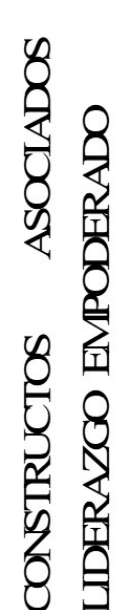 } & Creatividad & $57,67,71,13,3,59,25,38,8$ \\
\hline & $\begin{array}{l}\text { Proceso participativo de toma de } \\
\text { decisiones }\end{array}$ & $11,24,10,12,20$ \\
\hline & Auto Liderazgo & 60 \\
\hline & Motivación & 53,15 \\
\hline & LMX & $27,28,32,39$ \\
\hline & Autoeficacia & $14,37,61,16,26,30$ \\
\hline & Satisfacción Profesional & 19 \\
\hline & Éxito Profesional & 3 \\
\hline & Interdependencia & 18 \\
\hline & Ambiente Proactivo & 45 \\
\hline & Visibilidad de la Tarea & 36. \\
\hline
\end{tabular}

Fuente: elaboración propia.

pendiente otras variables (Zhang y Gheibi, 2015). Recientemente (Lee, Willis, y Tian, 2017) realizan un meta análisis sobre liderazgo empoderado y recapitulan sobre los ambientes que lo favorecen, retoman el proceso de definición, destacando la importancia del enfoque en la tarea y los ambientes facilitadores para el empoderamiento, sintetizando los hallazgos de las últimas décadas.

Aun cuando algunos de los instrumentos para evaluar el constructo son utilizados con mayor frecuencia, no se ha realizado la validación de este en otras poblaciones. Finalmente, destaca el hecho de no haber estudios que mencionen o realicen un seguimiento en la evaluación o evolución del liderazgo empoderado utilizando una segunda fase en la investigación, los estudios comparativos se realizan principalmente en poblaciones diferentes (Biemann, Kearney, y Marggraf, 2015; Harris, Li, Boswell, Zhang, y Xie, 2013; Park, Kim, Yoon, y Joo, 2016; Zhu y Chen, 2014) lo anterior, refleja una imagen estática sobre un fenómeno dinámico como lo es el liderazgo.

\section{Conclusión}

Los estudios analizados presentan gran variabilidad en relación a las poblaciones estudiadas, así como a los constructos asociados al liderazgo empoderado considerados por los investigadores, lo que deja un vacío en la continuidad, así como en la profundidad del abordaje sobre LE. Si bien él LE puede presentarse en cualquier población, en áreas organizacionales diferentes, y en contextos culturales diversos, que lleva a considerar al LE como un factor de utilidad para cualquier organización, sin embargo, no se concluye sobre las características de los involucrados ni los contextos en los que se desarrolla. Geográficamente se realiza la mayor investigación sobre liderazgo en Asia donde las características culturales son específicas, aun cuando en Estado Unidos las investigaciones son escasas destacan por ser metodológicamente innovadoras y diferentes por el tipo de intervención, utilizando modelos de simuladores, o con intercambio de poblaciones, lo que hace los resultados obtenidos más interesantes.

Las poblaciones de análisis aun cuando son diversas, en campos diferentes, los investigadores se enfocan hacia la posición jerárquica de los sujetos de estudio, solo un estudio toma en cuenta el tiempo de relación entre el líder y el seguidor, dejando abierta la cuestión de si el tiempo de la interrelación entre líder/seguidor constituye un factor determinante en el éxito o fracaso del liderazgo empoderado.

La diversidad en las características de las investigaciones complica comprender de forma holística como el constructo se manifiesta y evoluciona dentro de una organización. Es interesante 
que la mayoría de los resultados encontrados tengan como resultado un efecto positivo sobre la organización, el líder y el seguidor, desde múltiples perspectivas. Únicamente dos artículos mencionan los efectos menos favorables del liderazgo empoderado, siendo escuetos en su descripción.

Destaca el empleo de más de 5 instrumentos de evaluación, persistiendo en la construcción y propuesta de otras escalas, dejando abierta la opción de requerirse una alternativa mejor que mida, de manera objetiva el constructo. Finalmente es necesario rediseñar las metodologías con el objetivo aportar profundidad a la investigación.

Durante las últimas dos décadas el liderazgo empoderado ha transitado asociado a diversas variables psicológicas, sin embargo, estas solo se evalúan de forma transversal sin dar seguimiento en el tiempo a la evolución del constructo, a los cambios dentro de los equipos de trabajo, a la intensidad del constructo en los sujetos, la permanencia de los efectos en la organización así como el impacto de los cambios externos sobre el constructo para ambas partes que lo integran, dejando inconcluso si él LE favorece al otro constructo o viceversa por lo tanto queda pendiente para futuras investigaciones.

\section{Referencias}

Ahearne Michael, Mathieu John, Rapp Adam. (2005). To Empower or Not to Empower Your Sales Force? An Empirical Examination of the Influence of Leadership Empowerment Behavior on Customer Satisfaction and Performance. Journal of Applied Psychology, 90(5), 945-955. https://doi.org/10.1037/002 1-9010.90.5.945

Amundsen Stein y Martinsen Øyvind L. Martinsen. (2014a). Selfother agreement in empowering leadership: Relationships with leader effectiveness and subordinates' job satisfaction and turnover intention. Leadership Quarterly, 25(4), 784-800. https://doi.org/10.1016/j.leaqua.2014.04.007

Amundsen Stein y Martinsen Øyvind L. Martinsen. (2014b). Empowering leadership: Construct clarification, conceptualization, and validation of a new scale. Leadership Quarterly, 25(3), 487-511. https://doi.org/10.1016/j.leaqua.2013.11.009

Amundsen Stein y Martinsen Øyvind L. Martinsen. (2015). Linking Empowering Leadership to Job Satisfaction, Work Effort, and Creativity. Journal of Leadership \& Organizational Studies, 22(3), 304-323. https://doi.org/10.1177/1548051814565819

Arnold Josh A., Arad Sharon, Rhoades Jonathan A. y Drasgow Fritz. (2000) The empowering leadership questionnaire: the construction and validation of a new scale for measuring leader behaviors. J. Organiz. Behav. 21, 249-269

Audenaert Mieke y Decramer Adelien. (2016). When empowering leadership fosters creative performance: The role of problem-solving demands and creative personality. Journal of Management and Organization, 1-15. https://doi.org/10.1017/jmo.2016.20

Baker G. Ross (Commission on L. and M. in the N. (2011). The roles of leaders in high-performing health care systems. Commission on Leadership and Management in the NHS. The Kings Fund, 1-27. https://doi.org/10.1016/j.amjmed.2012.04.024

Bester Janie, Stander Marius W. y Van Zyl Llewellyn E. (2015). Leadership empowering behaviour, psychological empowerment, organisational citizenship behaviours and turnover intention in a manufacturing division. SA Journal of Industrial Psychology, 41(1), 1-14. https://doi.org/10.4102/sajip.v41i1.1215

Biemann Torsten, Kearney Eric y Marggraf Kathrin. (2015). Empowering leadership and managers' career perceptions: Examining effects at both the individual and the team level. Leadership Quarterly, 26(5), 775-789. https://doi.org/10.1016/j.leaqua.2015.03.003

Byun Gukdo, Dai Ye, Lee Soojin y Kang Seung-Wan. (2016). When Does Empowering Leadership Enhance Employee Creativity ? a Three-Way Interaction Test. Social Behavior \& Personality: An International Journal, 44(9), 1555-1564. https://doi.org/10.2224/sbp.2016.44.9.1555

Cheong Minyoung, Spain Seth m., Yammarino Francis J. y Yun Seokhwa. (2016). Two faces of empowering leadership: Enabling and burdening. Leadership Quarterly, 27(4), 602-616. https://doi.org/10.1016/j.leaqua.2016.01.006

Enterking Judith, Robb Elizabeth y Mclaren Susan. (2013). Clinical leadership for high-quality care: Developing future ward leaders. Journal of Nursing Management, 21(2), 206-216. https://doi.org/10.1111/j.1365-2834.2012.01408.x

Erkutlu Hakan y Chafra James. (2015). Empowering Leadership and Organizational Job Embeddedness: The Moderating Roles of Task Interdependence and Organizational Politics. Procedia - Social and Behavioral Sciences, 210, 3-10. https://doi.org/10.1016/j.sbspro.2015.11.321

Fong Kai Hung, Snape Ed. (2015). Empowering leadership, psychological empowerment and employee outcomes: Testing a multi-level mediating model. British Journal of Management, 26(1), 126-138. https://doi.org/10.1111/1467-8551.12048

Furley John, Cleland Jennifer, Del Mar Chris, Hanratty Barbara, Kadam Umesh, Lasserson Daniel, McCowan Colin, Magin Parker, Mitchell Caroline, Qureshi Nadeem, Rait Greta, Steel Nick, van Driel Mieke y Ward Alison. (2008). 
Leaders, leadership and future primary care clinical research. BMC Family Practice, 9(1), 52. https://doi.org/10.1186/1471-2296-9-52

Gkorezis Panagiotis. (2016). Principal empowering leadership and teacher innovative behavior: a moderated mediation model. International Journal of Educational Management, 30(6), 1030-1044. https://doi.org/10.1 108/IJEM-08-2015-0 113

Hao Po, He Wei y Long Li-Rong. (2017). Why and When Empowering Leadership Has Different Effects on Employee Work Performance: The Pivotal Roles of Passion for Work and Role Breadth Self-Efficacy. Journal of Leadership \& Organizational Studies, 154805181770751 . https://doi.org/10.1177/1548051817707517

Harris Brad T., Li Ning, Boswell Wendy R., Zhang Xin-an y Xie Zhitao. (2013). Getting What's New from Newcomers: Empowering Leadership, Creativity, and Adjustment in the Socialization Context. Personnel Psychology, 567-604. https://doi.org/10.1111/peps. 12053

Hassan Abbas, Ali Abdul y Huraja Khali, Hussein. (2016). Effect of the empowerment leadership's on job involvement reinforcement through a mediator role for strategic thinking skills. International Journal of Academic Research in Business and Social Sciences June, 6(6), 189-220. https://doi.org/10.6007/IJARBSS/v6-i6/2193

Ismail Azman, Mohamed Hasan Al-Banna, Sulaiman Ahmad Zaidi, Mohamad Mohd Hamran y Yusuf Munirah Hanim. (2011). An empirical study of the relationship between transformational leadership, empowerment and organizational commitment. Business and Economics Research Journal, 2(1), 89.

Kim Minseo y Beehr Terry A. (2017a). Directing our own careers, but getting help from empowering leaders. Career Development International, 22(3), 300-317. https://doi.org/10.1 108/CDI-1 1-2016-0202

Kim Minseo y Beehr Terry A. (2017b). Directing our own careers, but getting help from empowering leaders. Career Development International, 22(3), 300-317. https://doi.org/10.1 108/CDI-1 1-2016-0202

Kwak Won Jun y Jackson Christine L. (2015). Relationship building in empowering leadership processes: A test of mediation and moderation. Journal of Management and Organization, $\quad 21(4), \quad 369-387$. https://doi.org/10.1017/jmo.2015.11

Lee Allan, Willis Sara y Tian Amy Wei. (2017). Empowering leadership: A meta-analytic examination of incremental contribution, mediation, and moderation. Journal of
Organizational Behavior, (August 2016), 1-20 https://doi.org/10.1002/job.2220

Lee Soojin, Cheong Minyoung, Kim Myungsun y Yun Seokhwa. (2017). Never Too Much? The Curvilinear Relationship Between Empowering Leadership and Task Performance. Group \& Organization Management, 42(1), 11-38. https://doi.org/10.1177/1059601116646474

Lega Federico, Prenestini Anna y Rosso Matilde. (2017). Leadership research in healthcare: A realist review. Health Services Management Research, 30(2), 94-104. https://doi.org/10.1177/0951484817708915

Li Mingze, Liu Wenxing, Han Yi y Zhang Pengcheng. (2016). Linking empowering leadership and change-oriented organizational citizenship behavior. Journal of Organizational Change Management, 29(5), 732-750. https://doi.org/10.1108/JOCM-02-2015-0032

Li Mingze y Zhang Pengcheng. (2016). Stimulating learning by empowering leadership. Leadership \& Organization Development Journal, 37(8), 1168-1186. https://doi.org/10.1108/LODJ-01-2015-0007

Li Ning, Chiaburu Dan S. y Kirkman Bradley L. (2017). Cross-Level Influences of Empowering Leadership on Citizenship Behavior. Journal of Management, 43(4), 1076-1102 https://doi.org/10.1177/0149206314546193

Li Shao-Long, He Wei, Yam Kai Chi y Long Li-Rong. (2015). When and why empowering leadership increases followers??? taking charge: A multilevel examination in China. Asia Pacific Journal of Management, 32(3), 645-670. https://doi.org/10.1007/s 10490-0 15-9424-1

Liu Yingying. (2015). The Review of Empowerment Leadership. Open Journal of Business and Management Open Journal of Business and Manage-Ment, 3(3), 476-482. https://doi.org/10.4236/ojbm.2015.34049

Lorinkova Natalia m., Pearsall Matthew J. y Sims Henry P. (2013). Examining the Differential Longitudinal Performance of Directive Versus Empowering Leadership in Teams,Academy of Management. 56(2), 573-596.

Lorinkova Natalia M. y Perry Sara Jansen. (2017). When Is Empowerment Effective? The Role of Leader-Leader Exchange in Empowering Leadership, Cynicism, and Time Theft. Journal of Management, 43(5), 1631-1654. https://doi.org/10.1177/0149206314560411

McDermont Aoife, Kidney Rachel y Flood Patrick. (2011) Understanding leader development: learning from leaders. Leadership \& Organization Development Journal, 32(4), 358-378. https://doi.org/10.1108/01437731111134643

Özaralli Nurdan. (2015). Linking Empowering Leader to Creativity: 
The Moderating Role of Psychological (Felt) Empowerment. Procedia - Social and Behavioral Sciences, $\quad 181$ 366-376. https://doi.org/10.1016/j.sbspro.2015.04.899

Park, Jong Gyu, Kim, Jeong Sik, Yoon Seung Won y Joo, BaekKyoo. (2016). The effects of empowering leadership on psychological well-being and job engagement: the mediating role of psychological capital. Leadership \& Organization Development Journal, 38(3). https://doi.org/10.1108/JHTT-09-2016-0053

Parris, Denise Linda y Peachey, Jon Welty. (2013). A Systematic Literature Review of Servant Leadership Theory in Organizational Contexts. Journal of Business Ethics, 113(3), 377-393. https://doi.org/10.1007/s10551-0121322-6

Poirier Léandre-Alexis Chénard, Morin Alexandre J. S., y Boudrias, Jean-Sébastien. (2017). On the merits of coherent leadership empowerment behaviors: A mixture regression approach. Journal of Vocational Behavior. 103, part B: 66-75. https://doi.org/10.1016/j.jvb.2017.08.003

Praszkier Ryszard. (2015). Empowering Leadership: Embracing Endogenous Dynamics. Journal of Positive Management, 6(2), 34-58. https://doi.org/10.12775/JPM.2015.009

Schoeler Fausing Maj, Skriver Joensson Thomas, Lewandowski Joshua, y Bligh Michelle. (2009). Antecedents of shared leadership: Empowering leadership and interdependence. Emerald Insight, 29(5), 494-519. https://doi.org/10.1108/JFM-03-2013-0017

Sharma Payal Nangia y Kirkman Bradley L. (2015). Leveraging Leaders: A Literature Review and Future Lines of Inquiry for Empowering Leadership Research. Group \& Organization Management, 40(2), 193-237. https://doi.org/10.1177/1059601115574906

Srivastava Manjari y Vyas Ruta. (2015). Empowering leadership: A study of team leaders and team members. Indian Journal of Industrial Relations, 50(4), 712-717.

Tung Hui-Ling y Chang Yu-Hsuan. (2011). Effects of empowering leadership on performance in management team. Journal of Chinese Human Resources Management, $2(1), \quad 43-60$. https://doi.org/10.1108/20408001111148720

Wu Chi-Min y Chen Tso-Jen. (2015). Psychological contract fulfillment in the hotel workplace: Empowering leadership, knowledge exchange, and service performance. International Journal of Hospitality Management, $\quad 48, \quad 27-38$. https://doi.org/10.1016/j.ijhm.2015.04.008

Wong Humborstad Sut I., Nerstad Chistina G.L y Dysvik Anders. (2014). Empowering leadership, employee goal orientations and work performance. Personnel Review, 43(2), 246-271. https://doi.org/10.1108/PR-01-2012-0008

Zhang Pengcheng y Gheibi Samaneh. (2015). The gloomy picturesque empowering leadership through the lens of work engagement. European Scientific Journal, 11(10), 385-397.

Zhang Xiaomeng y Zhou Jing. (2014). Empowering leadership, uncertainty avoidance, trust, and employee creativity: Interaction effects and a mediating mechanism. Organizational Behavior and Human Decision Processes, $124(2)$, $150-164$ https://doi.org/10.1016/j.obhdp.2014.02.002

Zhu Yu-Qian y Chen Houn-Gee. (2014). Empowering Leadership in R\&D Teams: A Closer Look at the Process and Outcomes. The 8th International Conference on Knowledge Management in Organizations. Conference paper. 475-484. https://doi.org/10.1007/978-94-007-7287-8 


\section{ANEXO I \\ Relación de artículos revisados}

1

Arnold, J.A., Arad S., Rhoades J.A., and Drasgow F. of a new scale for measuring leader behavior

2000

000 The empowering leadership questionnaire: the construction and validation Journal of Organizational Behavior Abdul, A., \& Abbas, H. 2016 Effect of the empowerment leadership's on job involvement reinforcement through a mediator role for strategic thinking skills. International Journal of Academic Research in Business and Social Sciences Ahearne, M., Mathieu, J., \& Rapp, A. 2005

To Empower or Not to Empower Your Sales Force? An Empirical Examination of the Influence of Leadership Empowerment Behavior on Customer Satisfaction and Performance Journal of Applied Psychology 2011

The influence of empowering leadership, empowerment and engagement on affective commitment and turnover intentions in community health service workers: Test of a model. Leadership in Health Services Amundsen, S., \& Martinsen, Ø. L. effectiveness and subordinates' job satisfaction and turnover intention.

in empowering leadersh Amundsen, S., \& Martinsen, Ø.L. 2015

Empowering leadership: Construct clarification, conceptualization, and validation of a new scale Leadership Quarterly Amundsen, S; Martinsen, OL. 2015 The Role of Self-Leadership and Psychological Empowerment Journal of Leadership \& Organizational Studies Audenaert, M., \& Decramer, A. 2016 solving demands and creative personality

Linking Empowering Leadership to Job Satisfaction, Work Effort, and Creativity:

When empowering leadership fosters creative performance: The role of problemJournal of Management and Organization

Auh, S., Menguc, B., \& Jung, Y. S. $\quad 2014 \quad$ Unpacking the relationship between empowering leadership and service-oriented citizenship behaviors: a multilevel approach Journal of the Academy of Marketing Science

Bester, J; Stander, M. W; \& Van Zyl, L. E. $2015 \quad$ Leadership empowering behaviour, psychological empowerment, organisational citizenship behaviours and turnover intention in a manufacturing division Commission on Leadership and Management in the NHS. The Kings Fund

Biemann, T; Kearney, E; Marggraf, K. $2015 \quad$ Empowering leadership and managers' career perceptions: Examining effects at both the individual and the team level Leadership Quarterly

Bobbio, A., Bellan, M., \& Manganelli, A. M. 2012 Empowering leadership, perceived organizational support, trust, and job burnout for nurses: A study in an Italian general hospital. Health Care Management Review,

Byun, G Dai, Y; Lee, S ; Kang, SW. $2016 \quad$ When does empowering leadership enhance employee creativity? A three-way interaction test Social Behavior \& Personality: An International Journal

Carmeli, A; Schaubroeck, J; \& Tishler, A. $2011 \quad$ How CEO empowering leadership shapes top management team processes: Implications for firm performance The Leadership Quarterly

Chen, G., Sharma, P. N., Edinger, S. K., Shapiro, D. L., \& Farh, J. L. 2011 Motivating and demotivating forces in teams: crosslevel influences of empowering leadership and relationship conflict. Journal of Applied Psychology

Cheong, M; Spain, S.M; Yammarino, F.J; \& Yun, S. $2016 \quad$ Two faces of empowering leadership: Enabling and burdening Leadership Quarterly

Dewettinck, K., \& Van Ameijde, M. $2011 \quad$ Linking leadership empowerment behaviour to employee attitudes and behavioural intentions: Testing the mediating role of psychological empowerment. Personnel Review

Dierendonck, D., \& Dijkstra, M. 2012 The role of the follower in the relationship between empowering leadership and empowerment: A longitudinal investigation. Journal of Applied Social Psychology

Erkutlu, Hakan, Chafra, Jamel $2015 \quad$ Empowering Leadership and Organizational Job Embeddedness: The Moderating Roles of Task Interdependence and Organizational Politics Procedia-Social and Behavioral Sciences

Fausing, MS . Joensson TS. Lewandowski J. y Bligh M. 2015 Antecedents of shared leadership: empowering leadership and interdependence Leadership \& Organization Development Journal

Fong, K. H., \& Snape, E. $2015 \quad$ Empowering Leadership, Psychological Empowerment and Employee Outcomes: Testing a Multi level Mediating Model. British Journal of Management 
Gao, L., Jassen, O., Shi, K. 20 Leadership Quarterly

Gkorezis, P. $2016 \quad$ Principal empowering leadership and teacher innovative behavior: a moderated mediation model International Journal of Educational Management

Hao P., He W., and Long L. R. 2017 Why and When Empowering Leadership Has Different Effects on Employee Work Performance: The Pivotal Roles of Passion for Work and Role Breadth Self-Efficacy Journal of Leadership \& Organizational Studies

Harris, TB; Li, N; Boswell, WR; Zhang, XA; \& Xie, ZT. 2015 Getting what's new from newcomers: empowering leadership, creativity, and adjustment in the socialization context Personnel Psychology

Hassan A.A. \& Huraja H. $2016 \quad$ Effect of the empowerment leadership's on job involvement reinforcement through a mediator role for strategic thinking skills Personnel Psychology

Hassan, S. Mahsud, R. Yukl, G. \& Prussia, GE. (2013) $2013 \quad$ Ethical and empowering leadership and leader effectiveness Journal of Managerial Psychology

Hon, A. H.Y. and Chan W.W.H. 2012 Team Creative Performance: The Roles of Empowering Leadership, Creative-Related Motivation, and Task Interdependence Cornell Hospitality Quarterly

Kasemsap K. $2013 \quad$ Strategic Business Management: A Practical Framework and Causal Model of Empowering Leadership, Team Cohesion, Knowledge-Sharing Behavior, and Team Performance Journal of Social and Development Sciences

Kim M. y Beehr T.A. $2017 \quad$ Self-Efficacy and Psychological Ownership Mediate the Effects of Empowering Leadership on Both Good and Bad Employee Behaviors Journal of Leadership \& Organizational Studies

Kim M. y Beehr T.A. $2016 \quad$ Directing our own careers, but getting help from empowering leaders Career Development International

Kuo, R.Z \& Lee G.G. $2011 \quad$ Knowledge management system adoption: exploring the effects of empowering leadership, task-technology fit and compatibility, Behaviour \& Information Technology

Kuo, RZ., Lai M.F., \& Lee, G.G $2011 \quad$ The impact of empowering leadership for KMS adoption Management Decision

Kwak, WJ; Jackson, CL. 2017 Relationship building in empowering leadership processes: A test of mediation and moderationa Journal of Management and Organization

Lee, A., Willis, S., \& Tian, A. W. $2017 \quad$ Empowering leadership: A meta analytic examination of incremental contribution, mediation, and moderation. Journal of Organizational Behavior

Lee, S., Cheong, M., Kim, M., \& Yun, S. $2017 \quad$ Never too much? The curvilinear relationship between empowering leadership and task performance. $\quad$ Group \& Organization Management

Li S.L., \& He W. \& Yam K.C., \& Long R.L. $2015 \quad$ When and why empowering leadership increases followers' taking charge: A multilevel examination in China Journal of Organizational Change Management,

Li M. y Zhang P. (2016) $2016 \quad$ Stimulating learning by empowering leadership: Can we achieve cross-level creativity simultaneously? Leadership \& Organization Development Journal

Li, MZ; Liu, WX ; Han, Y ; \& Zhang, PC. $2016 \quad$ Linking empowering leadership and change-oriented organizational citizenship behavior The role of thriving at work and autonomy orientation Journal of Organizational Change Management

Li, N; Chiaburu, DS; Kirkman, BL. 2017 Cross-Level Influences of Empowering Leadership on Citizenship Behavior: Organizational Support Climate as a Double-Edged Sword Journal of Management

Liu, Y. 2015 The Review of Empowerment Leadership. Open Journal of Business and Management

Lorinkova, N. M., \& Perry, S. J. $2017 \quad$ When Is Empowerment Effective? The Role of Leader-Leader Exchange in Empowering Leadership, Cynicism, and Time Theft Journal of Management

Lorinkova, N. M., Pearsall, M. J., \& Sims, H. P. $2013 \quad$ Examining the differential longitudinal performance of directive versus empowering leadership in teams. Journal of Management

Magni, M., \& Maruping, L. M. $2013 \quad$ Sink or swim: Empowering leadership and overload in teams' ability to deal with the unexpected. Human Resource Management

Martin, SL; Liao, H; Campbell, EM $2013 \quad$ Directive versus Empowering leadership: a field experiment comparing impacts on task proficiency and proactivity Academy of Management Journal 
Martínez-Córcoles, M., Schöbel, M., Gracia, F. J., Tomás, I., \& Peiró, J. M. $2012 \quad$ Linking empowering leadership to safety participation in nuclear power plants: A structural equation model. Journal of safety research

McDermott, A., Kidney, R., \& Flood, P. 2011 Understanding leader development: learning from leaders. Leadership \& Organization Development Journal

Namasivayam, K; Guchait, P; Lei, P. $2014 \quad$ The influence of leader empowering behaviors and employee psychological empowerment on customer satisfaction International Journal of Contemporary Hospitality Management

Ozaralli N. $2015 \quad$ Linking Empowering Leader to Creativity: The Moderating Role of Psychological (Felt) Empowerment Procedia - Social and Behavioral Science

Pan, Q., \& Wei, H. 2009 Effects of Trust, Risk and Managerial Self-Efficacy on Leader Empowering Behavior. In Information Science and Engineering (ICISE)

Park,G, J., k Kim, J., Yoon, S. W., ... \& Joo, B. K. 2017 The effects of empowering leadership on psychological well-being and job engagement: The mediating role of psychological capital. Leadership \& Organization Development Journal

Poirier L.A.C., Morin A.J.S., Boudrias J.S. $2017 \quad$ On the merits of coherent leadership empowerment behaviors: A mixture regression approach Journal of Vocational Behavior

Praszkier R. $2015 \quad$ Empowering Leadership: embrasing endogenous dynamics Journal of Positive Management

Raub, S., \& Robert, C. $2010 \quad$ Differential effects of empowering leadership on in-role and extra-role employee behaviors: Exploring the role of psychological empowerment and power values. Human relations

Schoeler Fausing, M., Skriver Joensson, T., Lewandowski, J., \& Bligh, M. 2009 Antecedents of shared leadership: Empowering leadership and interdependence. Emerald Insight

Sharma, P.N; \& Kirkman, B.L. $2015 \quad$ Leveraging Leaders: A Literature Review and Future Lines of Inquiry for Empowering Leadership Research Group \& Organization Management,

Sla tten, T; Svensson, G; Sværi S. $2011 \quad$ "Empowering leadership and the influence of a humorous work climate on service employees' creativity and innovative behaviour in frontline service jobs" International Journal of Quality and Service Sciences

Srivastava, M., \& Vyas, R. $2016 \quad$ Empowering leadership: a study of team leaders \& team members. Indian Journal of Industrial Relations

Tekleab, A. G., Sims H. P. Jr., Yun, S., Tesluk, P. E. y Cox, J. 2008 Are we on the same page? Effects of self-awareness of empowering and trasformational leadership Journal of Leadership \& Organizational Studies

Tuckey, M. R., Bakker, A. B., \& Dollard, M. F. 2012 Empowering leaders optimize working conditions for engagement: a multilevel study. Journal of occupational health psychology

Tung, H. L. 2014 When empowering leadership links to team work outcomes: Encouraging the expression of psychological empowerment and knowledge sharing. GSTF Journal of Psychology

Tung, H. L., \& Chang, Y. H. 2011 Effects of empowering leadership on performance in management team: Mediating effects of knowledge sharing and team cohesion. Journal of Chinese Human Resources Management

Van Dijke, M., De Cremer, D., Mayer, D. M., \& Van Quaquebeke, N. 2012 When does procedural fairness promote organizational citizenship behavior? Integrating empowering leadership types in relational justice models. Organizational Behavior and Human Decision Processes

Vecchio, R. P., Justin, J. E., \& Pearce, C. L. $2010 \quad$ Empowering leadership: An examination of mediating mechanisms within a hierarchical structure. The Leadership Quarterly

Wong Humborstad S.I., Nerstad C. G.L., Dysvik A. 2014 Empowering leadership, employee goal orientations and work performance: A competing hypothesis approach Personnel Review,

Wua C.M., Y Chenb T.J $2015 \quad$ Psychological contract fulfillment in the hotel workplace: Empowering leadership, knowledge exchange, and service performance International Journal of Hospitality Management

Xue, Y., Bradley, J., \& Liang, H. $2011 \quad$ Team climate, empowering leadership, and knowledge sharing. Journal of knowledge management

Zhang, X., \& Bartol, K. M. $2010 \quad$ Linking empowering leadership and employee creativity: The influence of psychological empowerment, intrinsic motivation, and creative process engagement. Academy of management journal

Zhang P., Gheibi S. $\quad 2015 \quad$ The impact of empowering leadership on work performance and work family conflict: the role 


DOXAL

of gender European Scientific Journal European Scientific Journal mediating mechanism Organizational Behavior and Human Decision Processes mechanism. In Management Science and Engineering (ICMSE)

Empowering leadership in R\&D teams: a closer look at its components, process, and outcomes R\&D Management 